\title{
Using a free colour vision simulator to improve the accessibility of orienteering maps
}

\author{
Rebecca Schulga *, David Forrest, Trevor Hoey \\ School of Geographical and Earth Sciences, University of Glasgow, Glasgow, UK \\ Rebecca.Schulga@glasgow.ac.uk \\ David.Forrest@glasgow.ac.uk \\ Trevor.Hoey@glasgow.ac.uk \\ * Corresponding author
}

Keywords: Orienteering, Colour Vision Deficiency, Colour Vision Simulation, Map Specification Abstract:

Orienteering maps must be clear and readable due to demands on competitors to accurately navigate under time pressure. Colour is used to indicate runnability (ease of traversing through terrain) and distinguish between features, many of which would be omitted from other types of topographic mapping. Use of colour can be a barrier to participation for the $8 \%$ of men and $0.4 \%$ of women who have colour vision deficiencies (CVD), commonly known as 'colour blindness' (Birch, 1993). Map use by those with CVD is lower than in the general population (Olson and Brewer, 1997) partly due to frustrations differentiating between map symbols which appear identical or may not even be visible.

Colours on orienteering maps are controlled by international specifications (the International Specification for Orienteering Maps, ISOM 2017 (IOF Map Commission, 2017) and the International Specification for Sprint Orienteering Maps, ISSOM 2006 (IOF Map Commission, 2006)) and chosen to be unambiguous to those with CVD. However, Long and Junghans (2008) found that some of the specified colours could be easily confused by people with common types of CVD. Additionally, maps for lower levels of competition are often made by club members who have little or no formal cartographic training. Specifications are frequently used incorrectly, and printers rarely calibrated with the official test sheet. Mapper inexperience and issues with the underlying specifications combine to give a poor experience to orienteers (or potential orienteers) with CVD.

In a preliminary investigation into the accessibility of orienteering maps, four experienced orienteers with CVD were interviewed about the use of colour on both ISOM and ISSOM orienteering maps. Based on issues they reported, four versions of one ISOM map were produced to test alternative colours for certain symbols. Participants were asked to rank the four map images in order of readability. The map images were then input into Coblis, a free, online colour vision simulator (https://www.color-blindness.com/coblis-color-blindness-simulator/). The outputs from Coblis were ranked by people with no CVD. Correlation between how images were ranked indicates that Coblis produces a useful simulation of what is seen by those with CVD and is suitable for informing the design of orienteering maps. It is suggested that orienteering mappers should adopt this or a similar tool to check and improve accessibility of maps before printing. Colour vision simulators could also contribute to a larger review of map specifications for accessibility, including developing ways to modify individual maps for competitors with rare vision anomalies.

Table 1 shows the four map images as they were created, and a simulation of how they would appear to a person with a deuteran anomaly - an inability to detect green light - the most common type of CVD (Long and Junghans, 2008). 'A' shows a map drawn to specification, while ' $\mathrm{B}$ ', ' $\mathrm{C}$ ', and ' $\mathrm{D}$ ' test two variations for the colours of the course overprint and the map areas designated "out of bounds". All participants (those with CVD looking at the original images, and those without looking at the simulated images) stated that 'D' was the easiest to read. 


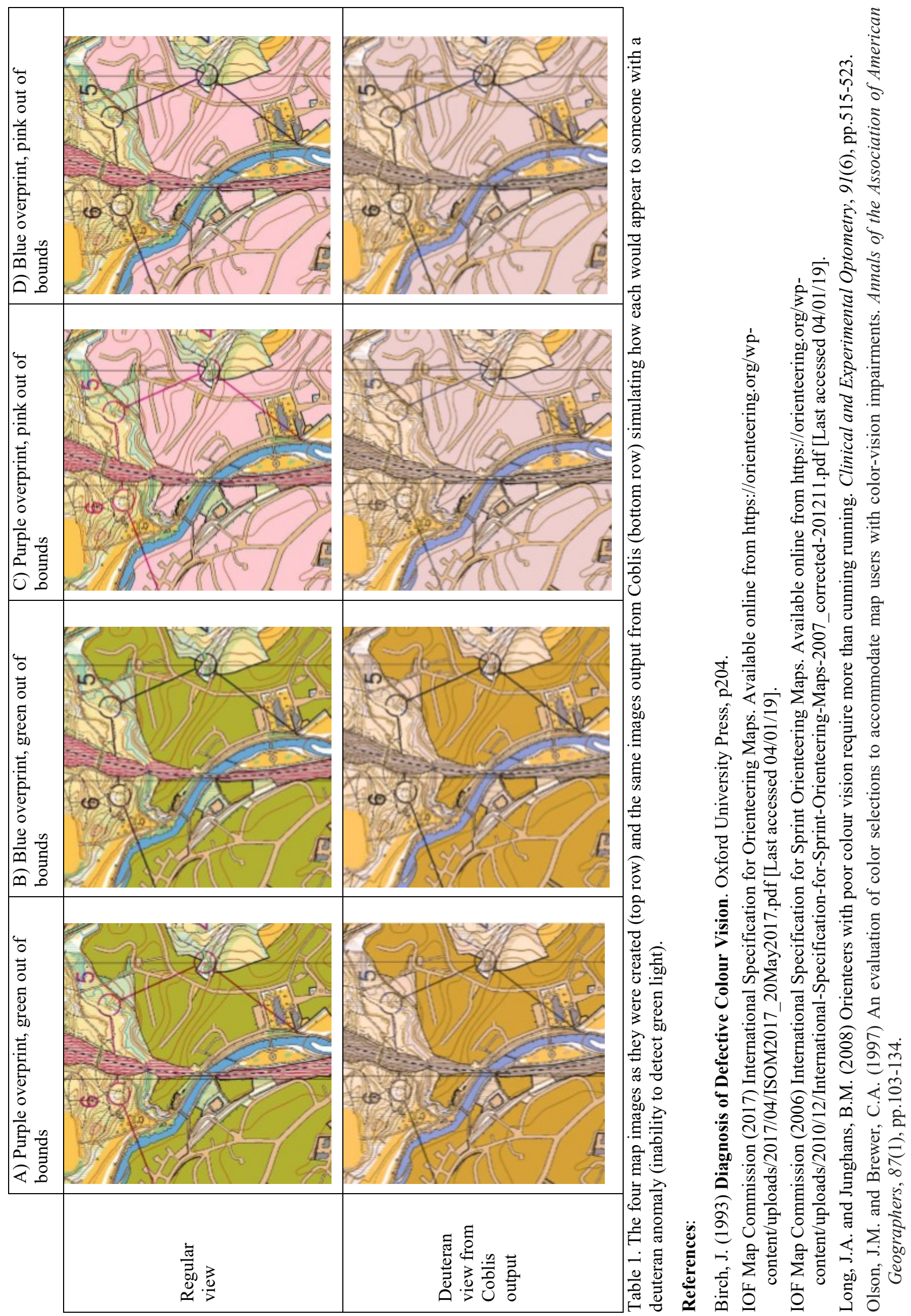

